\title{
Long Phase Coherence Time and Number Squeezing of two Bose-Einstein Condensates on an Atom Chip
}

\author{
G.-B. Jo, Y. Shin, S. Will, T. A. Pasquini, M. Saba, W. Ketterle, and D. E. PritchardÆ \\ MIT-Harvard Center for Ultracold Atoms, Research Laboratory of Electronics, \\ Department of Physics, Massachusetts Institute of Technology, Cambridge, MA 02139, USA \\ M. Vengalattore, M. Prentiss \\ MIT-Harvard Center for Ultracold Atoms, Jefferson Laboratory, \\ Physics Department, Harvard University, Cambridge, MA 02138, USA
}

(Dated: October 19, 2018)

\begin{abstract}
We measure the relative phase of two Bose-Einstein condensates confined in a radio frequency induced double well potential on an atom chip. We observe phase coherence between the separated condensates for times up to $\sim 200 \mathrm{~ms}$ after splitting, a factor of 10 longer than the phase diffusion time expected for a coherent state for our experimental conditions. The enhanced coherence time is attributed to number squeezing of the initial state by a factor of 10 . In addition, we demonstrate a rotationally sensitive (Sagnac) geometry for a guided atom interferometer by propagating the split condensates.
\end{abstract}

PACS numbers: 03.75.Dg, 39.20.+q, 03.75.-b, 03.75.Lm

Precision measurements in atomic physics are usually done at low atomic densities to avoid collisional shifts and dephasing. This applies to both atomic clocks and atom interferometers. At high density, the atomic interaction energy results in so-called clock shifts [1], and leads to phase diffusion in Bose-Einstein condensates (BECs) 2 2, 3, 4, 5, 6, 7]. Most precision measurements with neutral atoms are performed with free-falling atoms in atomic beams [8, 9] or in fountain geometries [10]. Major efforts are currently directed towards atom interferometry using confined geometries, such as atom traps or waveguides, often realized by using atom chips [11]. These geometries are promising in terms of compactness and portability, and also offer the prospect of extending interrogation times beyond the typical $0.5 \mathrm{~s}$ achievable in the atomic fountains [10].

However, given the deleterious effects of high atomic density, those devices were thought to be able to operate only at low density and therefore at small flux, seriously limiting the achievable signal-to-noise ratio and sensitivity. Here we show that we can operate BEC interferometer at high density, with mean field energies exceeding $h \times 6 \mathrm{kHz}$, where $h$ is Planck's constant. Using a radio frequency (RF) induced beam splitter [13, 14, 15], we demonstrate that condensates can be split reproducibly, so that even after $200 \mathrm{~ms}$, or more than one thousand cycles of the mean field evolution, the two condensates still have a controlled phase. The observed coherence time of $200 \mathrm{~ms}$ is ten times longer than the phase diffusion time for a coherent state. Therefore, repulsive interactions during the beam splitting process [16] have created a non-classical squeezed state with relative number fluctuations ten times smaller than for a Poissonian distribution.

Our work is a major advance in the coherence time of confined atom interferometers, which have operated at interrogation times below $\sim 50 \mathrm{~ms}$ [14, 17, 18] due to technical limitations. Our work also advances the preparation of number squeezed states to much higher atom numbers. Previous experiments in optical lattices [19, 20] and in an optical trap 21] were limited to very small populations ( $\sim 1-1000$ atoms). In addition, the fact that the clouds could be prepared on an atom chip with dc and RF electric currents, but without any laser beams, is promising for future applications. Finally, operating the RF induced beam splitter on propagating condensates, we realized an on-chip Sagnac interferometer.

For two separated Bose-Einstein condensates, a state of well-defined relative phase (phase coherent state), $|\phi\rangle$, is a superposition state of many relative number states, $\left|N_{r}=N_{1}-N_{2}\right\rangle$, where $N_{1}$ and $N_{2}$ are the occupation of each well for $N=N_{1}+N_{2}$ atoms. Because of atomatom interactions in the condensates, the energy of number states, $E\left(N_{1}, N_{2}\right)$, have quadratic dependence on the atom numbers $N_{1}$ and $N_{2}$ so that the different relative number states have different phase evolution rates [2, 5]. A superposition state will therefore have a spread of evolution rates, causing "phase diffusion" or "decoherence" of the relative phase with time. In contrast to normal diffusion processes, the phase uncertainty, $\Delta \phi$, increases here linearly. The phase diffusion rate, $R$, is proportional to the derivative of the chemical potential of condensates, $\mu\left(N_{i}\right)(i=1,2)$, with respect to the atom number and the standard deviation of the relative atom number, $\Delta N_{r}$ : $R=(2 \pi / h)\left(d \mu / d N_{i}\right)_{N_{i}=N / 2} \Delta N_{r}$ 2, 3, 3, 4, 5, 6, 7]. A number squeezed state with sub-Poissonian number fluctuations $\left(\Delta N_{r}=\sqrt{N} / s\right)$, where $s>1$ is the squeezing factor, will exhibit a reduced phase diffusion rate relative to a phase coherent state with $\Delta N_{r}=\sqrt{N}$.

Bose-Einstein condensates of $\sim 4 \times 10^{5}{ }^{23} \mathrm{Na}$ atoms in 
(a)

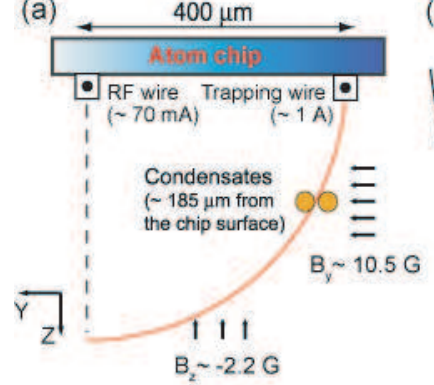

(b)
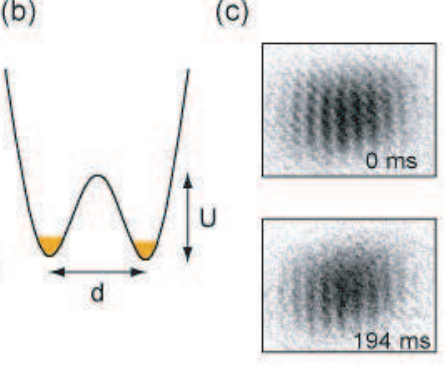

FIG. 1: Schematic of the atom chip interferometer. (a) Atoms were confined radially by the combined magnetic potential of a current-carrying wire and external bias field. Axial confinement in $x$ direction was provided by a pair of endcap wires (not shown) [12]. By dressing the atoms with an oscillating radio frequency $(\mathrm{RF})$ field from a second wire, the single minimum in the magnetic trapping potential was deformed into a double-well [14]. If the trapping position lies on the circle containing the trapping wire and centered on the RF wire, the splitting occurs in the horizontal plane. Condensates were placed $185 \mu \mathrm{m}$ away from the chip surface. For the single well, the radial (axial) trap frequency was $f_{r}=2.1 \mathrm{kHz}$ $\left(f_{z}=9 \mathrm{~Hz}\right)$ and the Larmor frequency at the trap center was $\approx 190 \mathrm{kHz}\left(B_{x} \approx 0.27 \mathrm{G}\right)$. Splitting was performed over $75 \mathrm{~ms}$ by linearly ramping the frequency of the RF field from $143 \mathrm{kHz}$ to $225 \mathrm{kHz}$. Gravity points in the $+z$ direction. (b) Double-well potential. The separation $d$ between the two wells and the barrier height $U$ were controlled by adjusting the frequency or amplitude of the RF field. (c) Matter wave interference. For various hold times after splitting, absorption images of condensates released from the double-well potential were taken after $10 \mathrm{~ms}$ time-of-flight. The field of view is $260 \times 200 \mu \mathrm{m}$.

the $\left|F=1, m_{F}=-1\right\rangle$ state were transferred into a magnetic trap generated by the trapping wire on an atom chip and external bias field [12]. A double-well potential in the horizontal plane was formed using adiabatic RFinduced splitting as described in Fig. 1a [13, 14]. Typically, the separation of the two wells was $d \sim 8.7 \mu \mathrm{m}$, the height of the trap barrier was $U \sim h \times 30 \mathrm{kHz}$, and the chemical potential of the condensates, measured from the trap bottom, was $\mu \sim h \times 6 \mathrm{kHz}$ (Fig. 1b). The lifetime of the atoms at the splitting position was $\sim 1.8 \mathrm{~s}$, significantly longer than in our previously demonstrated two-wire splitting method [12, 15, 22]. RF-induced splitting has several advantages over two-wire schemes: no loss channel (open port) during splitting, less sensitivity to magnetic bias fields, and realization of high trapfrequencies far from a surface [23]. Atoms were held in the double well for varying hold times, released by turning off the trapping potential within $30 \mu$ s at a known phase of the RF field [24], overlapped, and interfered in time-of-flight (Fig. 1c). The relative phase of the two condensates was measured as the spatial phase of the interference pattern [12, 17].

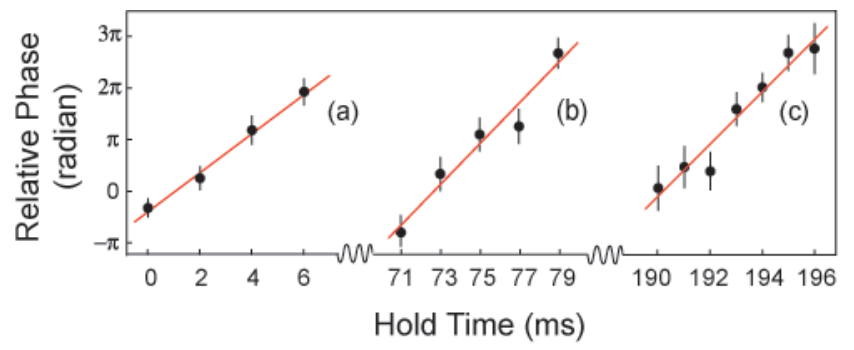

FIG. 2: Phase evolution of the relative phase during three different time intervals. The evolution rate of the relative phase are determined from the linear fit to be (a) $191 \mathrm{~Hz}$, (b) $198 \mathrm{~Hz}$, and (c) $255 \mathrm{~Hz}$. The data points represent the average of ten measurements for (a) and (b), and fifteen for (c).

An atom interferometer requires two independent condensates without any weak link which may lock the phase [12, 17]. To demonstrate this independence, we monitored the relative phase over short intervals right after and after up to $190 \mathrm{~ms}$ delay time. During each of these intervals, the phase evolved linearly with time at $\sim 2 \pi \times 200 \mathrm{~Hz}$, the signature of independent condensates (Fig. 2). The non-vanishing phase evolution rates are attributed to small asymmetries in the two trapping potentials, which lead to slightly different chemical potential after the splitting process. The time variation of this rate is attributed to axial motion of the two separated condensates. Note that the observed drift of the phase evolution rate of $\sim 60 \mathrm{~Hz}$ is only $1 \%$ of the condensates' chemical potential. In principle, the phase drift could be zeroed by a compensation field, but this has not been attempted.

To rule out the possibility that any weak link existed during the $200 \mathrm{~ms}$ time evolution and reset the relative phase, we demonstrated that an applied phase shift could be read out $200 \mathrm{~ms}$ after its appliance (Fig. 3). This proves that two independently evolving condensates have preserved phase coherence up to $200 \mathrm{~ms}$, a factor of 10 longer than the phase diffusion time, $\tau_{c}=1 / R \simeq 20 \mathrm{~ms}$, for our parameters.

For the quantitative study of phase fluctuations, the standard deviation of the phase does not provide the best characterization because the phase is measured modulo $2 \pi$. In the limit of a large data set, a completely random distribution has a phase variance of $\sim(3 \pi / 5)^{2}$. Already for smaller variances, the overlap of the tails of the Gaussian distribution can cause ambiguities. As a more appropriate measure of correlation, we represent each measurement of the relative phase as a phasor with unit length and compare the length of the sum of $N$ measured phasors with the expectation value of $\sqrt{N}$ for $N$ random phasors. The larger the difference, the smaller is the probability that the data set is compatible with a random phase distribution. This probability of uncorrelated 


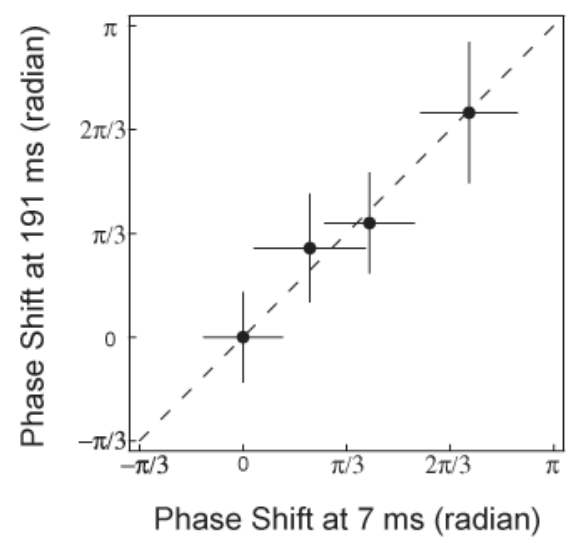

FIG. 3: Long phase coherence of two separated condensates. Applying various phase shifts to the condensates at $2 \mathrm{~ms}$ after splitting, the shifts of the relative phase were measured at $7 \mathrm{~ms}$ and $191 \mathrm{~ms}$, showing strong correlation. The dotted line denotes the ideal case of perfect phase coherence. Phase shifts were applied by pulsing an additional magnetic field in the $z$ direction for $50 \mu$ s with variable amplitude.

phases is called randomness 25]. Uncorrelated data have an expected value of randomness near 0.5 , while strongly correlated data would have a small value, e.g. ten data points drawn from a distribution with variance $(\pi / 5)^{2}$ have a probability of only $10^{-4}$ to be compatible with uncorrelated phases.

To study phase diffusion in our system, we analyze the distribution of ten measurements of the relative phase at various times after splitting, as shown in Fig. 4. The data show a well-defined phase (with probability $>90 \%$ ) for times shorter than $\sim 200 \mathrm{~ms}$. In contrast, the simulation for a coherent state in our experimental conditions, shown as a blue dotted line, predicts the same scatter of phase measurements already after $\sim 20 \mathrm{~ms}$. Fitting a phase diffusion model to the data points with randomness probability $>0.1$, gives a phase diffusion time of $200 \mathrm{~ms}$. The solid line is a fit which includes the initial variance $\Delta \phi_{0}^{2}$ :

$$
\Delta \phi(t)^{2}=\Delta \phi_{0}^{2}+(R t)^{2}
$$

The variance of the initial state, $\Delta \phi_{0}^{2}=(0.28 \pi)^{2}$ is dominated by technical noise including fitting errors and nonideal trap switch off. The contribution due to initial number fluctuations, $\Delta \phi_{0}^{2} \simeq\left(s / \Delta N_{r}\right)^{2}$, is $\sim\left(7.1 \times 10^{-4} \pi\right)^{2}$ for a coherent state $(s=1)$, and remains small unless the squeezing leads to number fluctuations on the order of a single atom, $s \sim \sqrt{N}$. The fitted value for the phase diffusion rate of $R=5 \mathrm{~s}^{-1}$ includes technical shot-to-shot variations in the relative atom number of two condensates after splitting and thermal fluctuation. Therefore, the inferred squeezing factor $s=10$ represents a lower bound. It implies that our relative atom number fluctuations were smaller than $\pm 0.03 \%$ corresponding to \pm 50 atoms.

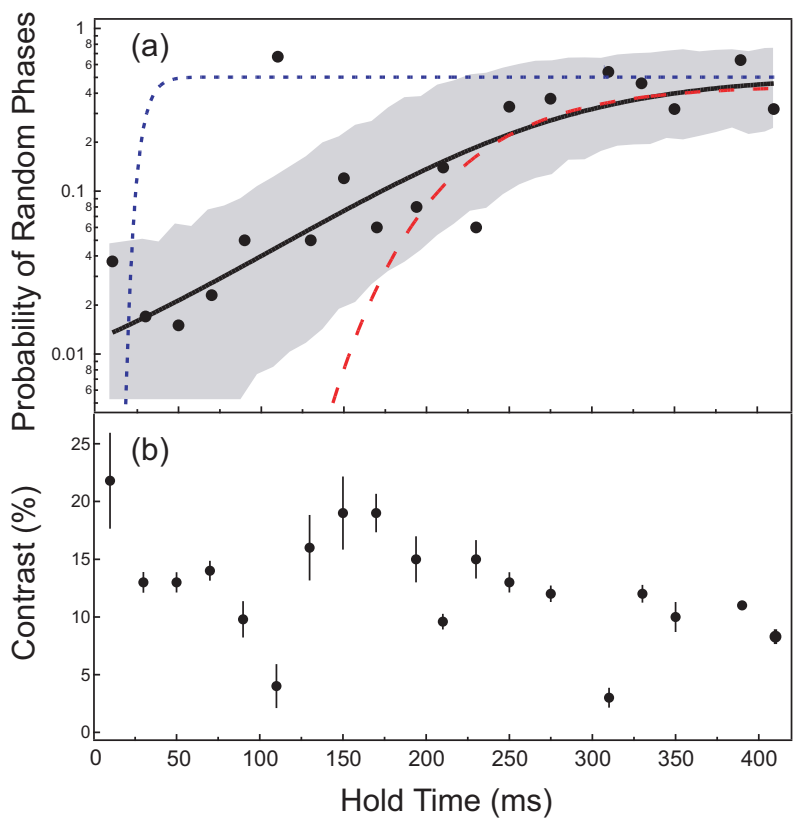

FIG. 4: Phase diffusion and number squeezing. (a) The randomness probability of ten measurements of the relative phase is displayed up to $400 \mathrm{~ms}$ after splitting. The blue dotted curve (red dashed curve) shows a simulation for a phasecoherent state (number squeezed state with $s=10$ ), which have negligible initial phase uncertainty. The solid line includes an initial phase uncertainty of $0.28 \pi$ (see text). The shaded region represents the window where ten data points from the sample with the given phase uncertainty would fall with $50 \%$ probability. (b) Contrast of the interference pattern. Since the endcap wires generate a field gradient $\frac{\partial B_{z}}{\partial x}$ as well as a field curvature $\frac{\partial^{2} B_{x}}{\partial x^{2}}$ at the position of the condensates, the two wells are not parallel to the trapping wire and consequently have slightly different axial trapping potential. This difference induces relative axial motion of the two condensates, which periodically reduces the contrast.

Locally the interference pattern of two pure condensates should always have $100 \%$ contrast, where contrast is defined as the density amplitude of the interference fringes over the mean density. Since in our experiment the contrast is derived from interference pattern integrated along the line-of-sight, it decreased gradually with time and exhibited fluctuations most likely due to asymmetric axial motion (Fig. 4b). Except for small regions near 110 and $300 \mathrm{~ms}$ hold time, the contrast was above $10 \%$, sufficient for accurate determination of the phase. The small windows with poor contrast have a large probability for random phases.

The observed long phase coherence time implies that the initial state is number squeezed. The probable origin of number squeezing during the splitting is repulsive atom-atom interactions [16]. The interactions make it energetically favorable for the two condensates to split with equal numbers in a symmetric double-well poten- 
(a)

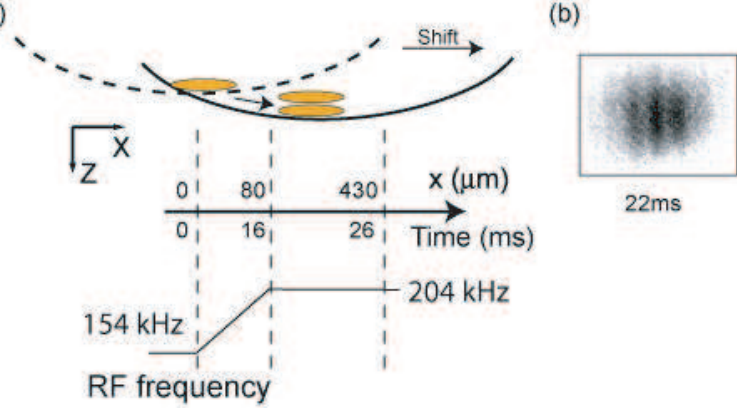

FIG. 5: Confined atom interferometry with enclosed area. (a) A single trapped condensate at rest was accelerated by shifting the trap center by $\sim 430 \mu \mathrm{m}$ in the axial direction $(t=0 \mathrm{~ms})$. The frequency of the RF field was linearly ramped from $154 \mathrm{kHz}$ to $204 \mathrm{kHz}$ during $16 \mathrm{~ms}$ after launch, splitting the condensates and separating them by $5-6 \mu \mathrm{m}$. (b) Phase measurements were done for up to $26 \mathrm{~ms}$ after the launch. The probability for random phases was determined for data sets of ten measurements. Until $16 \mathrm{~ms}$, this probability was extremely low (less than $10^{-12}$ ) and the relative phase was constant, implying that the two condensates were still connected. For $t>16 \mathrm{~ms}$, the relative phase evolved (similar to Fig. 2), and the probability for a random phase distribution was smaller than $10 \%$. This demonstrates that phase coherence was preserved after full splitting. The figure shows the interference pattern for $t=22 \mathrm{~ms}$. The field of view is $260 \times 200 \mu \mathrm{m}$.

tial, whereas number fluctuations, such as in a coherent state, cost energy. Describing splitting dynamics by the Josephson Hamiltonian shows how the interplay of tunneling and interactions leads to an increase of squeezing as the barrier is increased [16, 26]. Assuming that the squeezing can no longer increase when the Josephson frequency becomes slower than the inverse splitting time, we estimate a squeezing factor of $\sim 13$ for our experimental conditions [26]. For our elongated condensates, phase fluctuations are present for temperature above $\sim 100 \mathrm{nK}$ which is $\sim 1 / 10$ of the BEC transition temperature [27]. Since we cannot measure the temperature of an almost pure condensate in time of flight, it is not clear whether the interfering condensates had correlated phase fluctuations or not.

The work presented so far, and also all previous work on interferometry of confined or guided atoms, featured geometries without any enclosed area between the two paths of the interferometer. An enclosed area is necessary for rotational sensitivity [8, 9, 28] and requires moving atoms. As described in Fig. 5, we were able to extend the coherent beam splitter to condensates moving on an atom chip. The observed coherence time (10 ms) and propagation distance after splitting $(\sim 350 \mu \mathrm{m})$ were only limited by the chip geometry. This corresponds to an enclosed area of $\sim 1500 \mu \mathrm{m}^{2}$, and a response factor $\frac{4 \pi m A}{h} \sim 7.9 \times 10^{-5} \mathrm{rad} / \Omega_{e}$ for rotation sensing, where $m$ is the probe particle mass and $\Omega_{e}$ the earth rotation rate [29].

In conclusion, the present work demonstrates a long phase coherence time of $\sim 200$ ms between two spatially separated condensates on an atom chip, rivaling the interrogation times in fountain-type interferometers [10]. Number squeezing by a factor $\geq 10$ occurs during the preparation of the split state, providing a well-defined phase beyond the phase diffusion limit for a coherent state. Thus, interaction-induced squeezing reduces the phase diffusion caused by the same interactions [16]. These results show that it is both possible and promising to use condensates at high density for interferometry on an atom chip.

This work was funded by DARPA, NSF, ONR, and NASA. G.-B. Jo and S. Will acknowledge additional support from the Samsung Scholarship and the Studienstiftung des deutschen Volkes, respectively. We thank C.Christensen for experimental assistance.

* URL: http://cua.mit.edu/ketterle_group/

[1] K. Gibble and S. Chu, Phys. Rev. Lett 70, 1771 (1993).

[2] Y. Castin and J. Dalibard, Phys. Rev. A 55, 4330 (1997).

[3] M. Lewenstein and L. You, Phys. Rev. Lett. 77, 3489 (1996).

[4] E.M. Wright, D.F. Walls, and J.C. Garrison, Phys. Rev. Lett. 77, 2158 (1996).

[5] J. Javanainen and M. Wilkens, Phys. Rev. Lett. 78, 4675 (1997).

[6] A.J. Leggett and F. Sols, Phys. Rev. Lett. 81, 1344 (1998).

[7] J. Javanainen and M. Wilkens, Phys. Rev. Lett. 81, 1345 (1998).

[8] A. Lenef et al., Phys. Rev. Lett. 78, 760 (1997).

[9] T.L. Gustavson, P. Bouyer, and M.A. Kasevich, Phys. Rev. Lett. 78, 2046 (1997).

[10] A. Peters, K. Chung, and S. Chu, Nature 400, 849 (1999).

[11] F. Folman et al., Advances in atoimc, molecular, and optical physics 48, 263 (2002).

[12] Y. Shin et al., Phys. Rev. A 72, 021604(R) (2005).

[13] O. Zobay and B.M. Garraway, Phys. Rev. Lett. 86, 1195 (2001).

[14] T. Schumm et al., Nature physics 1, 57 (2005).

[15] Y. Colombe et al., Europhys. Lett. 67, 593 (2004).

[16] C. Menotti et al., Phys. Rev. A 63, 023601 (2001).

[17] Y. Shin et al., Phys. Rev. Lett. 92, 050405 (2004).

[18] O. Garcia et al., Phys. Rev. A 74, 031601(R) (2006).

[19] C. Orzel et al., Science 291, 2386 (2001).

[20] M. Greiner et al., Nature 415, 39 (2002).

[21] C.-S. Chuu et al., Phys. Rev. Lett. 95, 260403 (2005).

[22] E.A. Hinds, C.J. Vale, and M.G. Boshier, Phys. Rev. Lett. 86, 1462 (2001).

[23] I. Lesanovsky et al., Phys. Rev. A 73, 033619 (2006).

[24] We observed strong correlation between the population distribution of the spin components and the phase of the $\mathrm{RF}$ field at the moment of release, probably since the strength of the RF field ( $\sim 0.35 \mathrm{G})$ was comparable to the 
local static field $(\sim 0.27 \mathrm{G})$ [30]. The trapping potential was switched off at a value of the RF phase chosen to release atoms predominantly in the $\left|F=1, m_{F}=-1\right\rangle$ state.

[25] N. Fisher, Statistical analysis of circular data (Cambridge University Press, New York, 1993).

[26] A. Burkov , M. Lukin and E. Demler, unpublished
[27] D.S. Petrov, G.V. Shlyapnikov, and J.T.M. Walraven, Phys. Rev. Lett. 87, 050404 (2001).

[28] M. Sagnac, C.R.Acad. Sci 157, 708 (1913).

[29] P. Berman, Atom Interferometry (Academic Press, New York, 1997).

[30] S. Autler and C. Townes, Phys. Rev. 100, 703 (1955). 